\title{
Beschaffung als strategische Funktion
}

\section{Liebe Leserinnen und Leser,}

von den drei Grundfunktionen eines Unternehmens - Absatz, Produktion und Beschaffung - kam letzterer in der Vergangenheit regelmäßig die geringste Aufmerksamkeit zu. Nicht mit der Aura des Kreativen umgeben wie das Marketing, nicht offensichtlich wertschaffend wie die Produktion, kann man die Beschaffung leicht als sehr operativ, als ein rein technisches Einkaufen (miss-)verstehen. Ein Controlling hierfür trägt starke Kontrollzüge, sei es gerichtet auf die Einhaltung von Standardprozessen, sei es zur Vermeidung von Vorteilsnahmen der Einkäufer.

Seit einiger Zeit ist dieses Bild allerdings (endlich) ins Wanken gekommen. Unternehmen müssen sich immer mehr auf ihre Kernkompetenzen konzentrieren und verstärkt auf Dritte vertrauen, auf die sie zurückgreifen können. Sie sind nicht mehr in der Lage, für alles und jedes Blaupausen zu erstellen und/oder eine hohe Wertschöpfungstiefe wirtschaftlich aufrechtzuerhalten. Lieferanten-Kunden-Beziehungen bekommen häufiger einen relationalen Charakter; die Innovation findet beim Lieferanten statt, der sie dem Kunden in der Anwendung erschließt. Die Beschaffung entfernt sich damit zunehmend vom klassischen Einkauf und wird mehr und mehr zu einer Funktion, die die Verfügbarkeit strategischer Ressourcen sicherstellt.

Wollen Controller in diesem neuen Umfeld ihrer BusinessPartner-Rolle gerecht werden, dürfen sie auf der einen Seite die Effizienzreserven im Einkauf nicht aus den Augen verlieren. Hier steht nicht zuletzt durch das Thema Digitalisierung von Einkaufsprozessen ein erheblicher Veränderungsdruck ins Haus. Auf der anderen Seite müssen die Controller aber auch die Entwicklung der Beschaffung nachvollziehen. Dies führt zu neuen Fragestellungen, insbesondere auf dem strategischen Spielfeld. Ausgangspunkt ist die Frage, ob und wie weitgehend die Wertschöpfungstiefe des Unternehmens reduziert werden soll. Aspekte der Finanzierbarkeit von eigenen Fähigkeiten, die dem State of the Art entsprechen, sind in Ausgleich mit möglichen Abhängigkeiten von Dritten zu bringen. Es schließt sich die Auswahl derjenigen Kompetenzen an, die strategisch auch weiterhin „inhouse“ vorhanden sein sollen (Kernkompetenzen). Im dritten Schritt sind die

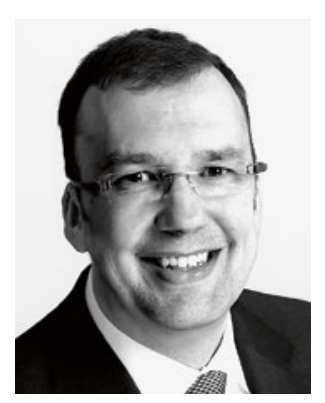

Utz Schäffer

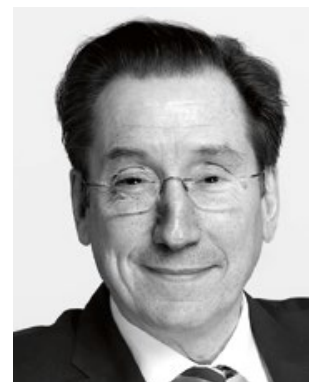

Jürgen Weber strategischen Partner festzulegen. Hierbei geht es unter anderem um eine ausgewogene Machtstruktur und um ähnliche Unternehmenskulturen.

Themen dieser Art sind Controllern bislang nur selten geläufig. Sie lassen sich nicht direkt in finanziellen Größen ausdrücken. Auf solche ist der Beitrag der Controller in der strategischen Steuerung aber heute noch konzentriert. Die Beschaffung liefert ein weiteres Beispiel dafür, dass Controller gerade im strategischen Bereich noch tiefer in die Geschäftsmodelle und Geschäftsprozesse des Unternehmens eindringen müssen. Vergleichsweise weit gekommen sind sie dabei im Vertrieb - das WHU Controller Panel zeigt hier eine deutliche Entwicklung über die letzten Jahre. Für die Beschaffung gilt dies aber nicht, ebenso wenig für die - damit eng verbundene - Logistik beziehungsweise das Supply Chain Management. Hier besteht noch Raum für Verbesserungen. Erst wenn die Controller in der strategischen Steuerung auch inhaltliche Beiträge für die Festlegung der Funktionalstrategien leisten können, also materiell helfen, zu besseren Strategien zu kommen, werden sie ihrem Anspruch als Business Partner gerecht.

Viel Vergnügen bei der Lektüre wünschen Ihnen
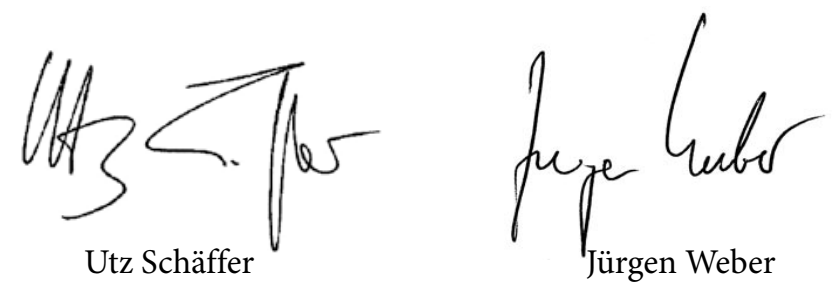